TWENTY-FIVE YEARS OF LINGUISTICS AND PHILOSOPHY

\title{
1. Philosophy And Linguistics And Linguistics and Philosophy
}

\subsection{Apology}

A BibTeX-formatted bibliography of the first twenty-five years of Linguistics and Philosophy, with topic keywords, is available at www.eecs.umich.edu $/ \sim$ rthomaso/l-and-p/. Rather than attempting an overview of what has been accomplished in the pages of this journal, or even a proper introduction to the papers in this issue that celebrate its twentyfifth anniversary, we instead try to present our own view of why each of the two subjects is profoundly and enduringly important to the other. The lack of italics in the title is intentional: we are concerned here more with Linguistics and Philosophy than with Linguistics and Philosophy, although of course the two are related. Our discussion begins with a general rationale, which we illustrate with two case studies, dealing with natural language metaphysics and with some philosophical issues in the semantics of words. The case studies are meant to provide examples of the sort of interesting, nontrivial interactions between Linguistics and Philosophy that create a special need and niche for Linguistics and Philosophy. They illustrate the many, and many unexpected, ways that the two fields affect one another. The role of L\&P in fostering this interaction has been enormous and salutary for both fields.

\subsection{The Importance of Linguistics to Philosophy}

To begin with, philosophy consists in using common sense intuitions to attack ill-defined, important, fundamental problems: the basic categories of existence and change, the nature of human thought and knowledge, and the ultimate goals of human life. The perennial questions of philosophy have a foundational bias - they are directly related to intuitions that are accessible to all of us, they do not use any specialized methods, and they often have an aporemic or dialectical flavor. 
But such problems can lead to specialized methods and questions, and as these develop and become more circumscribed, separate, technical disciplines may emerge. The foundations of these disciplines will typically repeat, in more refined form, the perennial unsolved questions of philosophy. But the bulk of the "normal science" in which unphilosophical specialties are engaged uses empirical and theoretical methods that have developed far from the original, unrefined methods of philosophy.

Although it has always maintained contact with immediate, common sense methodology, professional philosophy has also developed specialized methods of its own. Methods that depend on insights into language and linguistic usage have been used in philosophy since Plato and Aristotle. But these linguistic techniques became especially important in Twentieth Century philosophy. Cassirer (1955), Carnap (1956) and Austin (1956-57) all published at about the same time, ${ }^{1}$ illustrate three very different influences of linguistic methods on philosophy. Cassirer, a neoKantian, felt that the best evidence concerning the fundamental categories of human cognition came from linguistic anthropology. Cassirer's work therefore combines the Kantian tradition in philosophy with the methods of linguists like Wilhelm von Humboldt and Otto Jesperson. Carnap, on the other hand, represents the tradition in philosophical logic, which combines intuitions concerning language and reasoning with the methods of symbolic logic that emerged during the late Nineteenth and early Twentieth Century. And Austin represents the "ordinary language" tradition of preparing the way for philosophical work by carefully and systematically examining linguistic usage.

Cassirer's project (which we would now characterize as that of recovering the basic categories of human thought by investigating universals of human language) was not continued as such in philosophy, though there has been a later (and more or less independent) revival of similar ideas in "natural language metaphysics" (Bach 1989). Related themes are pursued in later linguistic work in lexical semantics, and in the philosophical and linguistic work on events and event typology influenced by Zeno Vendler and Donald Davidson; see, for instance, Dowty (1979, 1988); Levin (1993), Parsons (1990) and Steedman (1998). However, unlike Cassirer's work, the traditions that Carnap and Austin represent were absorbed into the mainstream tradition in analytical philosophy that continues to dominate the English-speaking world to this day.

Neither Carnap's nor Austin's work relied directly on the results or methods of linguistics. Although some philosophers were influenced in

1 Cassirer (1955) had appeared in German during the 1920s. The 1955 publication is the English translation. 
their approach to language by structuralist linguistics and behaviorist psychology, contributions like Morris (1946), when viewed in retrospect, do not seem as significant as those that were inspired by cognitivist, postChomskian linguistics. Although one suspects that Austin would have found this linguistic work congenial, and though his regular Saturday morning groups devoted part of a term to reading Chomsky (1957), this took place only shortly before Austin's death, ${ }^{2}$ and there was no opportunity to see how this influence would have played out in his philosophy. C. G. New presents a convincing critique of the methods of ordinary language philosophy as practiced by Austin, concluding with the recommendation that these methods should be supplemented with "the techniques available in general linguistics for the handling of the linguistic material to be explained", ${ }^{3}$ but without, however, going into any detail about what these methods are or how they are to be deployed in philosophy. Much of the philosophical material published in L\&P provides useful case studies of how the techniques of post-Bloomfieldian linguistics can be used in this way; we will discuss several instances below.

Carnap's work, on the other hand, suggests a program of explicating constructions of natural language that do not belong to the narrowly mathematical vocabulary so successfully dealt with by symbolic logic. This program was in fact pursued incrementally by many subsequent philosophical logicians; Montague's work, which was heavily influenced by Carnap's ideas and methodology, systematizes much of this work, and makes the relation to English language syntax explicit. From this point on, the work of semantically minded linguists influenced by Montague and of philosophical logicians working in Carnap's tradition overlaps to a very considerable extent.

The foregoing history places linguistics in a special relationship to contemporary philosophy. Of course, the foundations of linguistics, like those of any science, provide material for philosophical reflection. But the results of linguistics - and especially the results of the branches of linguistics concerned with semantics - provide direct intuitions of the sort that have always served as the starting points of philosophical positions, and often provide them in a refined and relatively novel form. To many analytic philosophers, linguistics is a resource rather than a subject matter.

This relationship is not unique; for instance, recent ethical theory has been heavily influenced by ideas from economics, and many areas of contemporary philosophy are influenced by evolutionary theory. But the influence of linguistic methods is more direct and pervasive than the

\footnotetext{
2 See Warnock (1969, p. 15).

3 New (1966, p. 164).
} 
importation of technical ideas from sciences such as economics or evolutionary biology, since the methods of some areas of linguistics seem to provide premises very like those that have been traditionally used in approaching large-scale, perennial philosophical problems.

\subsection{The Importance of Philosophy to Linguistics}

Contemporary linguistics, and especially the parts of it that relate to the lexicon, sentence structure, and meaning, remains close to its philosophical foundations; many linguists working in these areas find it difficult to avoid involvement in problems that are essentially philosophical, especially in trying to determine the cognitive basis of their work. Most of the cognitive sciences remain close to their philosophical roots, but linguists who have inherited the essentially introspective methods of generative grammar find themselves in an unusually philosophical position, since the research program is unsupported by a well developed experimental methodology.

Thus, the foundations of linguistics, and especially their relation to human cognition, have preoccupied linguists as much as or even more than philosophers. From Chomsky (1966) to Chomsky (2000), Noam Chomsky's work has represented an attempt to provide a philosophical account of the parts of linguistic science that can be grounded in the cognitive constitutions of individual human beings.

It is hard to find examples of philosophical foundations for a science, whether these are provided by professional philosophers or by the practitioners of the sciences themselves, that are broadly accepted by either community. The foundational questions raised by any successful science are difficult, and it is challenging to provide an account of the scientific results and practices that does justice to the topic, and that gives the reflective working scientist a sense of assurance in stepping back from the details of research to a larger view of its goals and methodology. The challenge is even greater in the cognitive sciences, which are closer to their foundations and in which it is difficult to arrive at a workable separation of philosophical from scientific problems.

L\&P has been less concerned with these foundational issues than with interactions between philosophy and the areas of linguistics concerned with semantics and pragmatics. These areas have seen many fruitful importations of ideas from philosophy into linguistic theory. The rich linguistic literature concerning eventualities and aspectual types was imported from a tradition in philosophy going back to Aristotle; likewise, so-called "donkey anaphora" 4 can be traced to Scholastic logic. Many

\footnotetext{
4 Including the donkey metaphor itself, which goes back to medieval times; see Ordine (1996a).
} 
of the more important recent themes in the theory of discourse, including implicature, presupposition, and the theory of focus phenomena, are realized in a literature that is heavily influenced by linguistic and philosophical ideas, and in which it is often impossible to disentangle the two components. All of these interactions are amply illustrated in the pages of L\&P.

\subsection{The Mission of $L \& P$}

The first issue of L\&P appeared in 1977, four years after the publication of Richard Montague's "Proper Treatment" (Montague 1973a) provided a new research focus for semantically minded linguists and linguistically minded philosophical logicians. The editorial statement (Wall 1977) in that issue points out that the journal will focus on "those areas where linguistic and philosophical studies overlap", and cites the following specific examples of such areas.

1. Traditional areas in the philosophy of language such as meaning and truth, reference, description, entailment, speech acts.

2. Traditional areas of linguistics such as syntax, semantics, and pragmatics (when the studies are aimed at shedding light on the fundamental nature of language).

3. Systems of logic with strong connections to natural language: modal logic, tense logic, epistemic logic, intensional logic.

4. Philosophical questions raised by linguistics as a science: linguistic methodology, the status of linguistic theories, the nature of linguistic universals.

5. Philosophically interesting questions at the intersection of linguistics and other disciplines: language acquisition, language and perception, language as a social convention.

This list of areas was repeated in the inside cover of the first issue. Over the years this statement has been amended. Some changes have been significant (such as the addition of some psycholinguistic and computational topics), but the strong focus on the intersection of linguistics and philosophy remains. It is not surprising, then, that, over the years, the articles published in L\&P have focused on this interdisciplinary area. But a healthy journal with such a focus would not have been possible without the persistence, over several academic generations, of a strong research group working in the intersection of the two fields. As in 1977, there remains today a group who identify their research with the intersection of linguistics and philosophy; L\&P has provided a sense of community for these people, an ongoing exhibit of the best work in the intersection of the 
fields, and - at its twenty-fifth anniversary - it has compiled a valuable archive of constructive cooperation between philosophers and linguistics.

\section{Natural Language Metaphysics: The Case of Mass and COUNT NOUNS}

Strawson (1959) and Cassirer (1955) took positions on the nature of language and thought that were in many ways similar, especially through their heavy influence by Kant; but they differed on one crucial point - Cassirer thought that the underlying structure of human thought could and should be investigated by looking to cross-cultural and anthropological studies of human cultures. He also favored the study of evolutionary development of societies, since he thought (departing in this from Kant) that this underlying structure evolved and that its different stages could be discerned by the study of the culture and language of these differing ("more primitive") societies. Strawson, on the other hand, had the view that all human thought was essentially the same, and that one could investigate this essential nature in 1950s Oxford as well as in other cultures.

As with Kant, the goal in both authors was to "lay bare" the underlying ways that we think and discourse about the world. Strawson called this "descriptive metaphysics":

Metaphysics has been often revisionary, and less often descriptive. Descriptive metaphysics is content to describe the actual structure of our thought about the world, revisionary metaphysics is concerned to produce a better structure ... we can distinguish broadly: Descartes, Leibniz, Berkeley are revisionary, Aristotle and Kant descriptive ... (Strawson 1959, p. xiii)

The details of the "actual structure of our thought about the world" that Strawson eventually described were to be justified by a psycho-semantic (conceptual) investigation.

... there is a massive central core of human thinking which has no history - or none recorded in histories of thought; there are categories and concepts which, in their most fundamental character, change not at all. Obviously these are not the specialities of the most refined thinking. They are the commonplaces of the least refined thinking; and are yet the indispensable core of the conceptual equipment of the most sophisticated human beings. It is with these, their interconnexions, and the structure that they form, that a descriptive metaphysics will be primarily concerned. (Strawson 1959, p. xiv)

But as Strawson saw it, these conceptual features had linguisticsemantic ramifications, and therefore to some degree at least we can determine the "actual structure of our thought" by an investigation into Language. It is never very clear in the writings of this school of "ordinary 
language philosophy" whether they believe that cross-linguistic studies will aid in the discovery of this structure. ${ }^{5}$ But in any case, Strawson thought that this investigation requires that we dig "beneath the surface of any natural language":

... when we ask how we use this or that expression, our answers, however revealing at a certain level, are apt to assume, and not expose, those general elements of structure which the metaphysician wants revealed. The structure he seeks does not readily display itself on the surface of language, but lies submerged. (Strawson 1959, p. xiv)

In Strawson's own assessment, the crucial point of this type of inquiry is that it can use a conceptual investigation to justify why our language works as it does. His own conceptual investigation "establish[ed] the central position which material bodies and persons occupy among particulars in general ... [I]n our conceptual scheme as it is, particulars of these two categories are the basic or fundamental particulars, ... other types of particulars must be seen as secondary" (p. xv). This discovery in turn "explain[s] the connexion between the idea of a particular in general and that of an object of reference or logical subject" (p. xvi), showing that these conceptual truths have a reflex in Language. Showing that our language works the way it does because it "mirrors" our conceptual scheme in turn justifies the "reverse" investigation of Language as a way to discover what our conceptual scheme is.

Strawson's own seminal work on language in Individuals was followed up by numerous writers intent on giving either (a) the "logical underpinnings" for some of Strawson's linguistic characterizations, or (b) the "metaphysical underpinnings" of these characterizations, or (c) further connections between these characterizations and other syntactico-semantic features of language.

We will review the history of one of Strawson's "key features of language", which has given rise to work in all three of these directions. This concerns his "sortal predication", which he contrasted with "featureplacing predication" and "characterizing predication", and the corresponding metaphysical distinction between "sortal universals", "feature-placing universals" and "characterizing universals".

A sortal universal is supposed to provide "a principle for distinguishing, counting, and re-identifying" individuals. The idea is that sortal predicates

\footnotetext{
5 Austin favored studying the way certain "experts" used ordinary language - for instance, judges and lexicographers. But he never advocated the study of speech acts as they are manifest in other languages, apparently thinking that they would be the same as in English. Strawson's work generated a rebuttal by Mei (1961), based on the contention that Chinese did not follow English in certain ways that Strawson assumed to be centrally important. Strawson apparently did not think this was interesting enough to respond to.
} 
correspond to reidentifiable particulars. Strawson feels that such particulars play a central role in our conceptual scheme. Sortal predicates contain, as part of their meaning, a way of counting and a way of re-identifying the particulars they denote. (See Gupta (1980) for one formalization of this idea.) To understand these terms is therefore to know whether "this particular of sort X is the same as that one", after it has undergone some change. It is to know, when confronted with some phenomenal experience, how to determine the number of particulars of that sort that there are in the experience.

The preeminance of particulars/individuals is a hallmark of Strawson's metaphysics; according to this view there is no such thing as a "bare individual", rather there are only "individuals under a (sortal) description". Strawson claims that even proper names presuppose an implicit sortal predicate.

A characterizing universal, on the other hand, presupposes that the individuals have already been distinguished; and it adds a further property to these individuals. While it is possible for a characterizing universal also to "offer a criterion of counting and grouping individuals", it only does so after the individuals have been distinguished by some antecedent means such as by a sortal universal.

Roughly, and with reservations, certain common nouns for particulars introduce sortal universals, while verbs and adjectives applicable to particulars introduce characterizing universals. (Strawson 1959, p. 170)

Strawson's final category is the feature-placing universals. These do not individuate particulars (as the sortal predicates do), nor do they characterize already-individuated particulars (as the characterizing predicates do). Strawson gives examples such as Snow is falling and There is water here, and says of them

Snow, water, coal and gold, for example, are general kinds of stuff, not properties or characteristics of particulars; though being made of snow or being made of gold are characteristics of particulars. Nor are the universal terms introduced into these propositions sortal universals. No one of them of itself provides a principle for distinguishing, enumerating and re-identifying particulars of a sort. (Strawson 1959, p. 208)

Strawson's metaphysical ideas led to the development of "sortal logics", which attempted to formalize the alleged primacy of sortals. Most of the theorists who developed these logics saw (as did Strawson himself) that sortal predicates naturally co-occurred with quantifiers, forming quantifier phrases like All people and Some tables. In turn this led to the development of logics for restricted quantification where the restrictions were combinations of quantifiers and sortal predicates. The proposed "logical form" of sentences like All people desire to learn was $[\forall x$ : 
Person $(x)$ ]Desire-to-learn $(x)$, which simultaneously displays the sortal predicate as having a distinguished syntactic position ${ }^{6}$ and illustrates how a quantifier "only makes sense" when it is combined with a restrictive noun to form a syntactic unit. ${ }^{7}$ This last feature, the representation of natural language quantified NPs as syntactic units in the representation language, is followed now by pretty much every theory in the formal linguisticsemantics literature. It is not accurate to say that this is due to the influence of these sortal logics on the linguistics literature, for Montague's writings provide an independent source. Nonetheless, some important writers (for example, James McCawley) were in fact influenced by these more strictly philosophical considerations to adopt restricted quantification in their semantic representations. ${ }^{8}$ There are various accounts of sortal logics now on the market, ${ }^{9}$ but it turns out to be quite difficult to describe a logic which honors the intuitive notion of sortal predicate, is not merely a syntactic variation on classical logic (such as ordinary restricted quantification), and yet is logically coherent.

It is natural for linguists and linguistically-oriented philosophers to identify Strawson's sortal predicates with count nouns, his feature-placing universals with mass nouns, and his characterizing universals with adjectives (and, following Strawson's own lead, with verbal elements). ${ }^{10}$ Just as Strawson had seen a special role for sortal predicates in language, so

6 On the assumption that happy is a characterizing predicate and that therefore happy person is not itself a complex sortal predicate, the representation of All happy people desire to learn would be

$$
[\forall x: \operatorname{Person}(x)](\operatorname{Happy}(x) \rightarrow \text { Desire-to-learn }(x)) .
$$

If (contrary to most Strawsonians) happy person were a complex sortal predicate, then it would be represented as

$$
[\forall x: \operatorname{Happy}(x) \& \operatorname{Person}(x)] \text { Desire-to-learn }(x) .
$$

7 The thought in many of these writers was that ordinary quantificational logic with its unrestricted quantifiers was committed to a doctrine of "bare particulars", where the values of the variables of quantification were merely "things" that have no qualities of their own and instead inherit whatever characteristics they have by means of figuring into the truth conditions of other parts of a sentence. Thus, in the example of the text, we would have $(\forall x)(\operatorname{Person}(x) \rightarrow$ Desire-to-learn $(x))$, which is to be read "For each $\mathrm{x}$, if $\mathrm{x}$ is a person then $\mathrm{x}$ desires to learn". But in this reading of the symbolic sentence it is seen that the "for each $\mathrm{x}$ " phrase presumes that $\mathrm{x}$ has no intrinsic qualities at all - it is a "bare particular" - but is awaiting the remainder of the sentence to see what types of properties are being asserted to hold.

8 There are also semantic accounts for "sortal incorrectness" either in or influenced by the Montague framework, e.g., (Thomason 1972; Waldo 1979; Keenan 1993).

9 Stevenson (1977) and Freund (2000) are two examples.

10 Although there are some clear differences: thing is a count noun, for instance, but not a sortal predicate. 
too did he see a special role for feature-placing predicates. And this led to a particular interest being paid to mass nouns, both from the point of view of metaphysics and from that of a logic capable of characterizing the special sorts of inferences in which mass terms can participate. Together, the metaphysical presuppositions and the logical characterizations can be seen to give rise to a formal semantics for mass terms. Pelletier (1974) surveyed the then-existing logical treatments of mass terms to show that some accommodation was needed, ${ }^{11}$ and Pelletier (1979) collected many works relating to the background metaphysics of mass terms (as well as some of the more strictly logical works). Fruitful deployments of this topic into computational linguistics (Bunt 1985) and formal semantics (Lønning 1987; Higginbotham 1994) followed, and work on the metaphysical implications of sortal and mass terms continues to appear (Lowe 1989, Pelletier 1991).

The pathway that started with Strawson's feature-placing universals, passing through the various metaphysical and logical issues that they evoke, and developing into computational accounts and formal semantic accounts of mass terms also had another rather surprising turn. The similarity of mass terms with plural count terms was noted, and this then led to a unified account of mass terms and plural count terms. This in turn generated accounts of distributivity and collectivity that can be grafted onto the account of plurals. ${ }^{12}$ This provides a striking case history of beneficial influences between Linguistics and Philosophy, in which each field provided the other with problems and projects, and which produced solutions that could not have been envisioned by either field alone.

Although Strawson himself regarded verbal elements as introducing characterizing universals, other writers noted similarities between linguistic-semantic features of the verbal system and the nominal system, especially as regards the count/mass distinction. Mourelatos (1977) related notions derived from observations of verbals ${ }^{13}$ to considerations derived from comparing mass with count nominals (such as divisiveness) to arrive at an elegant classification. Hoepelman and Rohrer (1980) drew parallels

\footnotetext{
11 For example, simple arguments like "This puddle is water, water is wet, so this puddle is wet" could not be validated in classical logical theories; statements like "water is water" could not be shown to be classical theorems. In fact, the whole underpinning of classical quantification theory seemed suspect: what could the values of $x$ be in quantified statements like $\forall x(M x \rightarrow G x)$, where $M$ is a mass term? "For each $x$, if $x$ is water ..." just seems to make no sense. There are also logical issues concerning the relationship between a mass term and the homophonous count term, for instance the relation between being liquid and being a liquid.

12 See Link (1983, 1998), Gillon (1992), Gil (1995), for example.

13 Notions such as events, achievements, processes, and the like; see Vendler (1957).
} 
between verbal tense and mass/count, and Bach (1981a) integrated this work into a more general theory of verbal and nominal types and their modifiers. Much recent linguistic work on "eventology" derives from these observations and analyses, and therefore depends, however distantly and indirectly, on Strawson's original work.

Metaphysics is the study of "what (sorts of) things there are". The Kantian view, emphasized by Strawson and Cassirer, is that we should look to our innate conceptual structure to determine what things we humans are forced as such to believe. And it is a short step from that inquiry to the question "What (sorts of) things do people talk as if there are?" To think that an answer to the latter question will yield an answer to the first question invokes a certain view about the nature of the faculty of Language and the structure of natural languages. If we take Strawson seriously and find that the sortal vs. feature-placing universal distinction marks a fundamental cleavage within human conceptual structure, then one will be chagrined to learn that different languages draw the count/mass distinction differently. Mei (1961) claimed that all simple nouns in (all of the dialects of) Chinese were mass, and that to form sortal predicates from them a speaker is required to employ a classifier phrase, much as English uses "drop of water". But then, says Mei, there is no primacy given to individuals that are introduced by sortal terms: everything ultimately depends on featureplacing universals. One can make this sort of point, of course, without referring to languages like Chinese. Even languages as closely related as English and French differ on various nouns: plate is count in English but la vasselle is mass in French, and dandruff is mass but les pellicules is count. Unless one wants to say that the innate conceptual structure of English speakers differs from that of French speakers, it seems clear that there can be no important conceptual feature that is univocally marked by the difference between mass and count terms. ${ }^{14}$

One might abandon universal relations between language and conceptual structure, and instead think of the metaphysics presumed just one natural language. Such an investigation - say, English language metaphysics - would seek to discover the sorts of things and relations among them that are needed to exhibit the structure of meanings that English seems to have. Instances of single-language metaphysics could then support a cross-

\footnotetext{
14 One might even wonder within one language, such as English, how there could be anything important to a distinction that put onions, beans, coins, shoes, beliefs, failures, noodles, and colds on one side while putting garlic, rice, change, footware, knowledge, success, spaghetti, and flu on the otherside. But we will not press this issue here. This train of thought would challenge the relation between language and thought, and would force us to reconsider the centrality of sortals and particulars.
} 
linguistic investigation of the features shared by all the various natural language metaphysics.

This single-language investigation is undertaken in Bach (1981, 1986a, b) which describe English metaphysics in detail. ${ }^{15}$ And Bach (1995a), written as the introduction to a systematic comparative of quantification in natural languages, is at pains to lay out the connections between studies of mass and count terms, studies of the interpretation of tense and aspect, and interpretation of quantification in the languages of the world. The central role assumed by the sortal/mass distinction and its analogical manifestation in the verbal system of English has become a central locus of investigation in some cross-linguistic studies (see Bach et al. 1995b, c).

Perhaps Strawson's insistence on the centrality of the sortal/mass distinction was correct, and the shortcoming was in his belief that the distinction was identically manifested in all languages because it was a universal feature of human cognition. Had he followed Cassirer instead, he might have allowed for the possibility that the distinction could evolve and transmogrify as Language and languages develop.

\section{LEXICAl SEMANTICS}

For most of its history, semantics has amounted to the study of the meanings of words. Except for studies of some limited word combinations - such as those arising from simple predications - even very ambitious earlier studies of semantics were confined to words. No doubt, the authors of these theories assumed that to explain the meaning of a phrase is simply a matter of explaining the meanings of its components.

As late a work as Ogden and Richards (1923) seems to equate meaning with the meaning of words; "grammar" is discussed in a brief appendix, which does not seem to raise the general issue of phrasal meaning. And even Katz and Fodor (1963) proposes a semantic rule that makes a sentence meaning no more than a sum of its word meanings.

The model-theoretic idea of assigning semantic values to phrases as a function of the values of their constituents, which was already well established in 1963, turns the assimilation of meanings to word meanings on its head. The general technique is masked by the syntactic simplicity of first-order-logic, but is evident in higher-order logic, especially in Alonzo Church's elegant formulations; see Church (1940). Here, words simply become the basis case of an inductive definition that assigns semantic values

\footnotetext{
15 Bach has also investigated the metaphysics of other natural languages, especially Pacific Northwest languages (Bach 1994, 1995).
} 
to phrases of arbitrary type. On this conception, the task of semantics is to interpret phrases: words, as semantically indecomposible phrases, are merely a degenerate special case.

This technique was imported into natural language semantics by Richard Montague, in papers dating from the late 1960s, all of them republished in Montague (1974). This work reinforced the idea that was already firmly in the minds of generative syntacticians, of the primacy of phrases for linguistic analysis, and of the crucial importance of explaining linguistic productivity. By now, it is one of the most generally accepted ideas across the various linguistic frameworks for doing semantics.

Although, strictly speaking, treating all categories as phrasal puts the meanings of words on the same footing as the meanings of other phrases of the same category, this approach has tended to produce a research paradigm that concentrates on the meanings of syntactic constructions, with attention paid to the meanings of specific words only when these meanings can be classified and studied by extending results from mathematical logic: natural language generalized quantifiers are one of these relatively rare cases. In many cases, these logical methods do not provide any way to distinguish the meanings of words, so different words tend to be treated as semantic primitives - different, but not different in any principled or specifiable way, so that the theory will make no distinctions between, say, talk' and sleep'. In practice, then, this approach exchanges the virtues and vices of its predecessor: concentrating on the meanings of constructions, there is difficulty in saying much about the basic units.

That being said, such an exchange does seem to improve our theoretical position. It was simply a mistake to assume that the meanings of phrases could be ignored, or that accounting for them would somehow be trivial. And now, words are explicitly present in contemporary semantic theories, so it is hardly possible to ignore them entirely. And it is not as if their meanings are in principle inexplicable; it is just that we lack logical methods for explicating them with the granularity that seems to be required.

Nothing, therefore, prevents us from hoping that an extended program of research, involving an empirical investigation of general features of word meaning and the development or importation from other areas of logical techniques for formalizing these features, would enable genuine progress in this area, from which a more adequate theoretical account of word meaning could emerge - an account which loses nothing that was learned in the meantime about phrasal meaning. One source of encouragement is that it seems to be possible to approach the matter incrementally. We certainly do not need to produce a complete definition (or componential analysis) of each word in our vocabulary. We can proceed "top down", 
adding partial content to words by means of meaning postulates; as the system of such postulates develops we may acquire much semantic information about individual words, even if full definitions are rare. Nothing prevents these postulates from being organized using an inheritance hierarchy, a technique that helps to provide a manageable organization of the lexicon in semantics as well as in other areas.

In fact, there are grounds for more than a mere hope. Progress in several different areas has produced results that contribute to this project; the challenge is to combine and further develop what has already been accomplished. The purpose of this section is to summarize some of this work and to indicate its relevance to the overall project.

\subsection{Derived Words}

A good way to begin this project is to remember that - even in morphologically impoverished languages like English - many words are structured in a way that bears on their meaning. The semantic constructions that we discover in this process also could prove to be useful in classifying words that do not appear to be morphologically complex. This area of study was mapped out in Dowty (1979), which identifies a number of important, recurring elements in semantically complex words. Unsurprisingly, these elements - becoming, causality, agency, and tending toward a culmination or goal - represent recurring themes in philosophy. This is not surprising; you would expect the semantic building-blocks of words to be important, recurrent ingredients of common sense.

Although these concepts have been an important part of philosophy ever since Aristotle, ${ }^{16}$ they have little or nothing to do with mathematics. In fact, one of the main achievements of the scientific work in the 17th century which replaced the Aristotelian picture of nature was to replace these common sense concepts with a very different theory, formulated in terms of a quantitative, continuous mathematics that dispenses with many of the important elements of the common sense picture of space, time, and change. The need to base mathematics on a foundation of rigorously proved theorems reinforced this departure from common sense.

Apparently, the important elements that are needed to formalize word meaning are connected with recognizable philosophical problems - often, problems that have a considerable history. And their formalization is problematic. It shouldn't be surprising that such concepts not only challenge

\footnotetext{
16 And their investigation in contemporary semantics owes a great deal to the philosophers who pointed out the relevance of Aristotle's ideas to linguistic problems, especially the problem of accounting for tense and aspect. See Vendler (1957), Kenny (1963) and Mourelatos (1977), for instance.
} 
philosophical reflection, but appear to resist formalization - at least, if a coherent model-theoretic semantics is a requirement, as Dowty assumed. We will briefly illustrate these points with becoming and causality.

Becoming. Dowty introduces a primitive BECOME in order to formalize the semantic derivation of inchoatives from statives - for instance, the derivation of darken from dark. The idea is that, where $p$ is function from temporal intervals and worlds to truth values, $\operatorname{BECOME}(p)(I)(w)$ is true at interval $I$ and world $w$ if and only if there are intervals $J$ and $K$ such that (1) $J$ overlaps $I$ and $I$ overlaps $K$ and (2) $p(J)(w)$ is false and $p(K)(w)$ is true. ( $I$ overlaps with $J$ if a final subinterval of $I$ has the same endpoints as an initial subinterval of $J$.) The idea is that a state comes into being over an interval in which it is false towards the beginning and true towards the end.

As formalizations of the difficult concepts involved in change and agency go, this one succeeds pretty well. It works well in many cases. But still, there are problems. As it stands, it does not deal with comparative inchoatives like worsen. Suppose that if an account of these is added, so that $x$ worsens holds of intervals in which the referent of $x$ is not worse (relative to some compared object, say $j$ ) towards the beginning, and is worse (relative to this same $j$ ) towards the end. The problem then arises that this combines with Dowty's rule for the progressive to yield the result that at most given moments the stock market is worsening and improving. We can state a related problem (one that Dowty noticed and discussed) in terms of an even more striking example; everyone's lifetime will be an interval of becoming dead, according to the definition.

Perhaps these difficulties are due to the loss of information in accounting for becoming over a stretch of time in terms of just the endpoints. But it is hard to identify the exact nature of the problem, since good intuitions are sparse about the truth conditions even of simple propositions over intervals. When Bennett and Partee (1982) introduced interval semantics, they tried to provide criteria to test for the truth-value of a proposition over an interval, but did not succeed in finding anything very robust. Despite the theoretical motivations for introducing intervals in attempting to explain the linguistic phenomena, which Bennett-Partee and Dowty document well, and the usefulness of intervals in cases like This seat is occupied, where the subinterval property is not satisfied, ${ }^{17}$ interval semantics forces us to assign values in many cases where there seems to be no very good criterion for assigning them, while at the same time

\footnotetext{
17 That is, This seat is occupied can be true over a stretch of time, even though This seat is not occupied is true over some subintervals of the larger interval.
} 
it seems to miss qualitative information that is an important part of our common-sense appreciation of change.

These early explorations of interval semantics can be seen as an attempt to meet the challenges of natural language metaphysics by a fairly conservatative and familiar sort of extension of the available logical apparatus. (It is a common mathematical generalization to extend a theory over a domain of objects to a theory over the domain of sets of these objects.) In contrast, the more recent event-based theories have better intuitive connections to the relevant phenomena; we can account for a darkening change, for instance, using an event structure in which a state in which darkness is absent is connected by a process of darkening to a state in which darkness is present. ${ }^{18}$

However, this approach creates a methodological gap that has not yet been successfully bridged. The current event-based semantics are not embedded in semantic "fragments" as general as those of the 1970s; the interactions between different constructions are not fully worked out, and the connection to logical theories - and hence, to reasoning - is less clear.

Causality. Causality, the other concept we will briefly examine, is far more exciting and problematic. But generally speaking, it presents a story that is similar to that of becoming.

Dowty (1979) was able to draw on attempts in philosophical logic to formalize the common sense notion of causality. He appeals to the theory of Lewis (1977), which in turn is based on the logic of conditionals presented in Lewis' earlier book (Lewis 1973). But again, there are flaws in the formalization, which have to be regarded not as simple errors in deploying the theory but as genuine problems. We mention a few of these.

The first problem has to do with the polymorphism of explicit causal verbs. For instance, causal make can take gerundive clauses in subject position, The stone's hitting the windshield made it crack, or ordinary noun phrases, The stone made the windshield crack. Like Lewis, Dowty takes the basic causal construction to be a relation between sentence types. Dowty's analysis of a sentence like Mary made John wash the car amounts to

(1) $\exists P\left[\operatorname{CAusE}\left({ }^{\wedge} P\left(\mathbf{M a r y}^{\prime}\right),{ }^{\wedge} \operatorname{wash}^{\prime}\left(\mathbf{J o h n}^{\prime}, a\right)\right]\right.$. (Dowty 1979, p. 225)

That is, there is a property such that Mary's having this property causes John to wash the car.

The difficulty is that if it happens to be true that anything at all - say John's loving to wash the car - made him wash the car, then, because

$$
\operatorname{love}^{\prime}\left(\mathbf{J o h n}^{\prime},{ }^{\wedge} \lambda x \operatorname{wash}^{\prime}(x, a)\right)
$$

18 See, for instance, Parsons (1990), Steedman (1998) and Higginbotham et al. (2000). 
is logically equivalent to

$$
\operatorname{Mary}^{\prime}=\operatorname{Mary}^{\prime} \& \operatorname{love}^{\prime}\left(\mathbf{J o h n}^{\prime},{ }^{\wedge} \lambda x \operatorname{wash}^{\prime}(x, a)\right),
$$

we can derive (1). This trick, which enables you to turn any proposition $p$ into a property that equivalently applies to John if and only if $p$, undermines Dowty's strategy for defining the CAUSE that takes nominal subjects in terms of the sentential subject CAUSE. The setback we have just noticed could either be just a minor annoyance or a sign of deeper problems in the whole approach to formalizing natural language causality.

The breadth and depth of recent contributions to causality and causal reasoning in disciplines including philosophy, statistics, and computer science provides a wealth of material for the project of introducing a formalization of causality into a linguistic theory. This literature is enormous; Lewis (1973), of course, is a philosophical landmark, but the subsequent philosophical literature on the topic is varied and extensive. ${ }^{19}$ Pearl (2000) uses ideas from the formalization of probabilistic reasoning in Artificial Intelligence, but is in fact an extended argument for the necessity of introducing explicit reasoning about causality into statistical formalisms. Pearl's approach, conceding that causality may not be an ingredient of basic physical theories or necessary for the reasoning processes of an unlimited intelligence, but stressing its vital importance for a limited intelligence interested in explaining and predicting in a complex world, fits well with the Kantian tradition and the goals of natural language metaphysics.

Attempts to formalize causality are also an important theme in other areas of Artificial Intelligence. This work goes back to the very beginnings of the field; one seminal paper, (Simon 1952) even predates the beginning of AI. Besides the work in AI connecting causality to statistical reasoning, there is a tradition, beginning with McCarthy and Hayes (1969) and McCarthy's earlier work, that seeks to understand the nature of common sense and common sense reasoning by formalizing it. This work is probably the most relevant to semantic concerns; some of it is concerned with general problems of causality and causal reasoning (see Ortiz 1999); some is concerned with "qualitative physics" or common sense reasoning about physical devices (see, for instance, Iwasaki and Simon 1986, Weld and de Kleer 1990); some is concerned with the role of causality in temporal reasoning, especially in planning and explanation (see, for instance, Lifschitz 1997).

\footnotetext{
19 The philosophical literature is voluminous, and we don't know of any recent article with a representative bibliography. For some references, see http://www.eecs.umich.edu/ rthomaso/bibs. References concerning causality can be found in the general bibliography by searching for "causality."
} 
These things make the study of common sense causality exciting, but do not make it easy. Even the theories that are well motivated and carefully worked out differ widely; and some (such as probability-based theories) would be difficult to integrate with the familiar formalisms used for natural language semantics.

The application of causality to natural language phenomena is complicated by the fact that lexical semantics makes some aspects of causality prominent that are not much studied in the general literature on causality. Many natural language causal constructions also involve immediacy (or perhaps normalcy); for instance, to say that Maude pounded the nail into the floor is not only to say that she caused the nail to be in the floor by pounding on it, but that the pounding caused the nail to go into the floor in the usual way. (The pounding couldn't, or couldn't normally, be a signal for someone else to pound the nail in.) Also, this causality seems to be contingent in many cases; for instance, to say that Stephen broke the goblet is not to say that what Stephen did to break the goblet must have broken it. He can't claim that he didn't break it by pointing out that he could have dropped it in the same way without its having broken. This consideration alone, if it is right, rules out most of the philosophical theories of causality.

Another vexed issue, which has been thoroughly canvassed in the literature subsequent to Dowty (1979), is the residual form of the "imperfective paradox"; exactly what is the relation between a true progressive sentence involving a telic verb, and the successful culmination of the corresponding process? Dowty's idea of an "inertial world" is very natural, and has the advantage of using standard ideas from possible worlds semantics. But the many objections to it are disturbing.

Eventuality semantics provides possible solutions to some of these problems. For instance, we may well be able to define causal constructions taking nominal subjects in terms of the AGENT role on events. We may even be able to dispense with causality, by using the relation between the initiating event that is part of a complex telic eventuality and the culminating state of that eventuality (when such a state exists) in place of it. $^{20}$

However, as before, there is a tradeoff. If we are to use eventuality semantics for purposes like this, we need to find ways to integrate eventualities into explicit semantic theories with linguistic coverage and rigor that is comparable to the classical formalisms that are based on possible worlds and higher-order logic alone.

\footnotetext{
20 See Steedman (1998) for background on complex eventualities. (The idea of using these eventualities in place of a causal primitive is not mentioned there, but is explored in Thomason (1999)).
} 


\subsection{The Semantic Classification of Words}

Reflection on linguistic meaning is challenged by the elusive nature of its relationship to linguistic form. A careful reading of Jespersen (1965) gives a forceful impression of the complexity of this relation; this impression is reinforced by the many examples put forward by Chomsky, from Chomsky (1975) to Chomsky (2000). Many philosophical projects during the last century seem in retrospect to have been misled in their conception by an overoptimistic conception of the simplicity of the relation between form and meaning. The idea that speech acts could provide an intermediary that would somehow close the gap between the sentence uttered and its meaning provides one such example; Grice's idea that the "logical" expressions of English in fact have the literal meanings suggested by logical theories could be another.

Such failures could induce wholesale skepticism concerning the viability of natural language semantics. ${ }^{21}$ But the possibility remains that this sense of failure may arise in the tendency of some philosophical programs to be overoptimistic and insufficiently empirical in approaching these problems, leading to oversimplified hypotheses, whereas the linguistic programs deriving from Montague's work are limited by what can be accomplished within the framework of Intensional Logic.

It is not impossible to find hypotheses concerning the relation of form and meaning that hold up much better under careful examination, and that also provide important insights into natural language metaphysics. Levin (1993) makes a very convincing case, based on extensive empirical evidence from many languages, that there are systematic correspondences between the patterns of argument structure that are associated with verbs and the semantic classification of the verb. The semantic categories that emerge from this investigation (for instance, change of state, changes that involve physical contact, changes involving locomotion) enlarge the semantic primitives discussed in Dowty (1979), and are clearly important ingredients in common sense reasoning about space, action, and change.

The formalization of such concepts seems to require the development of new logics. To a limited extent, there have been attempts in the linguistic literature to formalize some of the relevant ideas; for instance, see Dowty (1988, 1991). But cooperative efforts seem to offer the most promising avenue for progress in this area. Independently, researchers in Artificial Intelligence, and some philosophers, have been exploring issues that are very relevant to Levin's semantic categories; for work that deals with spatial

\footnotetext{
21 It is not difficult to find examples of this sort of skepticism; see, for instance, Schiffer (1987) and Chomsky (2000).
} 
representation and reasoning as well as dynamic issues, see Davis (1991), Stock (1997), Casati and Varzi (1999).

With this wealth of material to draw on, this seems to be one of the most exciting times for research in the intersection of linguistics and philosophy. We can surely took forward with anticipation to the next twenty-five additional years of $L \& P$.

\section{REFERENCES}

Austin, J. L.: 1956-57, 'A Plea for Excuses', Proceedings of the Aristotelian Society 57, $1-30$.

Bach, E., E. Jelinek, A. Kratzer, and B. Partee (eds.): 1995a, 'Introduction', in Quantification in Natural Languages, Vol. 1, pp. 1-11, Kluwer Academic Publishers, Dordrecht.

Bach, E., E. Jelinek, A. Kratzer, and B. Partee (eds.): 1995b, Quantification in Natural Languages, Vol. 1, volume 54 of Studies in Linguistics and Philosophy, Kluwer Academic Publishers, Dordrecht.

Bach, E., E. Jelinek, A. Kratzer, and B. Partee (eds.): 1995c, in Quantification in Natural Languages, Vol. 2, volume 55 of Studies in Linguistics and Philosophy, Kluwer Academic Publishers, Dordrecht.

Bach, E.: 1981, 'On Time, Tense, and Aspect: An Essay in English Metaphysics', in P. Cole (ed.), Radical Pragmatics, pp. 63-81, Academic Press, New York.

Bach, E.: 1986a, 'The Algebra of Events', Linguistics and Philosophy 9(1), 5-16.

Bach, E.: 1986b, 'Natural Language Metaphysics', in R. B. Marcus, G. J. W. Dorn, and P. Weingartner (eds.), Logic, Methodology, and Philosophy of Science, VII: Proceedings of the Seventh International Congress of Logic, Methodology, and Philosophy of Science, Salzburg, 1983, pp. 63-81, North-Holland, Amsterdam.

Bach, E.: 1989, Informal Lectures on Formal Semantics, State University of New York Press, Albany, NY

Bach, E.: 1994, 'The Semantics of Syntactic Categories', in J. Macnamara and G. E. Reyes (eds.), The Logical Foundations of Cognition, pp. 264-281, Oxford University Press, Oxford.

Bach, E.: 1995, 'A Note on Quantification and Blankets in Haisla', in E. Bach, E. Jelinek, A. Kratzer, and B. Partee (eds.), Quantification in Natural Languages, Vol. 1, pp. 13-20, Kluwer Academic Publishers, Dordrecht. In Bach et al. (1995b).

Bennett, M. R. and B. H. Partee: 1982, Toward the Logic of Tense and Aspect in English, Indiana University Linguistics Club, 310 Lindley Hall, Bloomington, Indiana.

Bunt, H. C.: 1985, Mass Terms and Model-Theoretic Semantics, Cambridge University Press, Cambridge, England.

Carnap, R.: 1956, Meaning and Necessity, Chicago University Press, Chicago, 2nd edition. (First edition published in 1947).

Casati, R. and A. C. Varzi: 1999, Parts and Places: The Structures of Spatial Representation, The MIT Press, Cambridge, MA.

Cassirer, E.: 1955, The Philosophy of Symbolic Forms, volume 1-3, Yale University Press, New Haven. (These works were first published (in German) in 1923-29).

Chomsky, N.: 1957, Syntactic Structures, Mouton, The Hague.

Chomsky, N.: 1966, Cartesian Linguistics, Harper and Row, New York. 
Chomsky, N.: 1975, 'Questions of Form and Interpretation', Linguistic Analysis 1(1), 75109.

Chomsky, N.: 2000, New Horizons in the Study of Language and Mind, Cambridge University Press, Cambridge, England.

Church, A.: 1940, 'A Formulation of the Simple Theory of Types', Journal of Symbolic Logic 5, 56-68.

Davis, E.: 1991, Common Sense Reasoning, Morgan Kaufmann, San Francisco.

Dowty, D. R.: 1979, Word Meaning in Montague Grammar, D. Reidel Publishing Co., Dordrecht, Holland.

Dowty, D.: 1988, 'On the Semantic Content of the Notion of "Thematic Role", in G. Chierchia, B. Partee, and R. Turner (eds.), Properties, Types and Meaning, Vol. 2, pp. 69-129, Kluwer Academic Publishers, Dordrecht.

Dowty, D.: 1991, 'Thematic Proto-Roles and Argument Selection', Language 67(3), 547619.

Freund, M.: 2000, 'A Complete and Consistent Formal System for Sortals', Studia Logica 65, 367-381.

Gil, D.: 1995, 'Universal Quantifiers and Distributivity', in E. Bach, E. Jelinek, A. Kratzer, and B. Partee (eds.), Quantification in Natural Languages, Vol. 1, pp. 321-362. Kluwer Academic Publishers, Dordrecht. (In Bach et al. 1995b).

Gillon, B.: 1992, 'Towards a Common Semantics for English Count and Mass Nouns', Linguistics and Philosophy 15(6), 537-639.

Gupta. A.: 1980, The Logic of Common Nouns, Yale University Press, New Haven, CN.

Higginbotham, J., F. Pianesi, and A. C. Varzi (eds.): 2000, Speaking of Events, Oxford University Press, Oxford.

Higginbotham, J.: 1994, 'Mass and Count Quantifiers', Linguistics and Philosophy 17(5), 447-480.

Hoepelman, J. and C. Rohrer: 1980, 'On the Mass-Count Distinction and the French Imparfait and Passé Simple', in C. Rohrer (ed.), Time, Tense, and Quantifiers: Proceedings of the Stuttgart Conference on the Logic of Tense and Quantification, pp. 629-645, Max Niemeyer Verlag, Tübingen.

Iwasaki, Y. and H. Simon: 1986, 'Causality in Device Behavior', Artificial Intelligence 29(1), 3-32.

Jespersen, O.: 1965, The Philosophy of Grammar, W. W. Norton, New York. (This work was originally published in 1924).

Katz, J. J. and J. A. Fodor: 1963, 'The Structure of a Semantic Theory', Language 39(2), $170-210$.

Keenan, E. L.: 1993, Natural Language, Sortal Reducibility, and Generalized Quantifiers', Journal of Symbolic Logic 58(1), 314-325.

Kenny, A.: 1963, Action, Emotion and the Will, Routledge and Kegan Paul, London.

Levin, B. C.: 1993, English Verb Classes and Alternations: A Preliminary Investigation, University of Chicago Press, Chicago, IL.

Lewis, D. K.: 1973, Counterfactuals, Harvard University Press, Cambridge, MA.

Lewis, D. K.: 1977, 'Causation', Journal of Philosophy 70(17), 566-567. Reprinted in D. K. Lewis, Philosophical Papers, vol. 2, Oxford University Press, 1986, pp. 159-213. (Postscripts are added in this reprinting).

Lifschitz, V.: 1997, 'On the Logic of Causal Explanation', Artificial Intelligence 96(2), $451-465$. 
Link, G.: 1983, 'The Logical Analysis of Plurals and Mass Terms: A Lattice-Theoretical Approach', in R. Bäuerle, C. Schwarze, and A. von Stechow (eds.), Meaning, Use, and Interpretation of Language, pp. 302-323, Walter de Gruyter, Berlin.

Link, G.: 1998, Algebraic Semantics in Language and Philosophy, CSLI Publications, Stanford, California.

Lønning, J. T.: 1987, 'Mass Terms and Quantification', Linguistics and Philosophy 10(1), $1-52$.

Lowe, E. J.: 1989, Kinds of Being: A Study of Individuation, Identity, and the Logic of Sortal Terms, Blackwell Publishers, Oxford.

McCarthy, J. and Hayes, P. J.: 1969, 'Some Philosophical Problems from the Standpoint of Artificial Intelligence', in B. Meltzer and D. Michie (eds.), Machine Intelligence 4, pp. 463-502, Edinburgh University Press, Edinburgh.

Mei, T.-L.: 1961, 'Subject and Predicate: A Grammatical Preliminary', The Philosophical Review 70(1), 153-175.

Montague, R.: 1973, 'The Proper Treatment of Quantification in Ordinary English', in J. Hintikka (ed.), Approaches to Natural Language: Proceedings of the 1970 Stanford Workshop on Grammar and Semantics, pp. 221-242, D. Reidel Publishing Co., Dordrecht, Holland, Reprinted in Montague (1974).

Montague, R.: 1974, Formal Philosophy: Selected Papers of Richard Montague, Yale University Press, New Haven, CN. Edited, with an introduction, by R. H. Thomason.

Morris, C. W.: 1946, Signs, Language and Behavior, Prentice-Hall, New York.

Mourelatos, A.: 1977, 'Events, Processes, and States', Linguistics and Philosophy 2(1), 415-434.

New, C. G.: 1966, 'A Plea for Linguistics', Mind 75, 368-384.

Ogden, C. K. and I. A. Richards: 1923, The Meaning of Meaning, Routledge \& Kegan Paul, London, 1st edition.

Ordine, N.: 1996, Giordano Bruno and the Philosophy of the Ass, Yale University Press, New Haven, CN.

Ortiz, Jr., C. L.: 1999, 'A Commonsense Language for Reasoning about Causation and Rational Action', Artificial Intelligence 111(1-2), 73-169.

Terence Parsons, T.: 1990, Events in the Semantics of English: A Study in Subatomic Semantics, The MIT Press, Cambridge, MA.

Pearl, J.: 2000, Causality: Models, Reasoning, and Inference, Cambridge University Press, Cambridge, England.

Pelletier, F. J.: 1974, 'On Some Proposals for the Semantics of Mass Nouns', Journal of Philosophical Logic 3(1-2), 87-108.

Pelletier, F. J. (ed.): 1979, Mass Terms: Some Philosophical Issues, D. Reidel Publishing Co., Dordrecht.

Pelletier, F. J.: 1991, 'Mass Terms', in H. Burkhardt and B. Smith (eds.), Handbook of Metaphysics and Ontology, pp. 495-499. Philosophia Verlag, Munich.

Schiffer, S.: 1987, Remnants of Meaning, The MIT Press, Cambridge, MA.

Simon, H.: 1952, 'On the Definition of the Causal Relation', The Journal of Philosophy 49, 517-528.

Steedman, M.: 1998, The Productions of Time. Unpublished manuscript, University of Edinburgh. Available from http://www.cogsci.ed.ac.uk/ steedman/papers.html.

Stevenson, L.: 1977, 'A Formal Theory of Sortal Quantification', Notre Dame Journal of Formal Logic 185-207, 185-207.

Stock, O. (ed.): 1997, Spatial and Temporal Reasoning, Kluwer Academic Publishers, Dordrecht. 
Strawson, P. F.: 1959, Individuals: An Essay in Descriptive Metaphysics, Methuen, London.

Thomason, R. H.: 1972, 'A Semantic Theory of Sortal Incorrectness', Journal of Philosophical Logic 2(2), 209-258.

Thomason, R. H.: 1999, Formalizing the Semantics of Derived Words, Unpublished manuscript, available at http://www.eecs.umich.edu/ $\sim$ rthomaso/documents.html.

Vendler, Z.: 1957, 'Verbs and Times', Philosophical Review 46, 143-160.

Waldo, J.: 1979, 'A PTQ Semantics for Sortal Incorrectness', in S. Davis and M. Mithun (eds.), Linguistics, Philosophy, and Montague Grammar, pp. 311-331, University of Texas Press, Austin, TX.

Wall, R.: 1977, 'Editorial Preface', Linguistics and Philosophy 1(1), 1-2.

Warnock, G. J. and J. L. Austin: 1969, 'A Biographical Sketch', in K. T. Fann (ed.), Symposium on J. L. Austin, pp 3-21, Routledge and Kegan Paul, London.

Weld, D. S. and J. de Kleer (eds.): Qualitative Reasoning about Physical Systems, Morgan Kaufmann, San Mateo, California.

Francis Jeffry Pelletier

Department of Philosophy

University of Alberta

Edmonton, Alberta

Canada T6G 2E5

E-mail: jeffp@cs.ualberta.ca

Richmond H. Thomason

Department of Philosophy

University of Michigan

Ann Arbor, Michigan

USA 48109-1003

E-mail: rich@thomason.org 
